\title{
Coherent thermal radiation of Fabry-Perot resonator structures
}

\author{
O.G. Kollyukh, V.P. Kyslyi, A.I. Liptuga, V. Morozhenko, V.I. Pipa, and E.F. Venger \\ V. Lashkaryov Institute of Semiconductor Physics, NAS of Ukraine \\ 41, prospect Nauky,03028Kyiv,Ukraine; e-mail:kollyukh@isp.kiev.ua
}

\begin{abstract}
The coherent thermal radiation from semiconductor plane-parallel resonator structures is investigated both theoretically and experimentally. The coherent properties of thermal radiation from these objects are manifested by sharp spectral lines and wellpronounced lobe-like directional patterns. We investigated the dependences of the intensities of spectral lines and the angular distribution of thermal radiation on optical parameters of the structures. New optical effects are presented, namely, the modulation of the coherent thermal radiation spectrum by an external magnetic field and a uniaxial pressure.
\end{abstract}

Keywords: thermal radiation, coherent radiation, plane-parallel structure, IR emitter.

Manuscript received 03.12.07; accepted for publication 19.12.07; published online 26.02.08.

\section{Introduction}

Thermal radiation (TR) emitted from heated solids is commonly viewed as incoherent radiation, i.e. that with the broad spectrum and a quasiisotropic angular distribution of the intensity. However, it has been demonstrated in a number of papers [1-11] that TR of some special sources possesses well-pronounced coherent properties. These are sharp spectral peaks (temporal coherence) and narrow angular lobes (spatial coherence). Coherent TR is generated via the following mechanisms: (i) excitation of surface waves (surface plasmons or surface phonon polaritons) that are coupled to propagating electromagnetic waves due to a surface grating [1-3]; (ii) wave interference effects in planeparallel structures [4-9]; and (iii) control of emissivity due to the existence of a photon band structure (photonic crystals [10-11]). The researches of coherent TR attracted a substantial interest due to its potential applications for the creation of novel controllable radiation sources intended for middle and far IR.

Here, we investigate the coherent TR of semiconductor plane-parallel resonator structures. Contrary to (i) and (iii) mechanisms (that may be responsible for the generation of coherent TR in some narrow spectral regions determined by the material properties), the interference effects in the resonator structures make it possible to develop coherent TR sources for wide spectral ranges. In addition, the structures studied by us have one more advantage: their manufacturing technology is easy.

We study the dependences of the spectral lines intensity and the angular distribution of TR on optical parameters of the structures. The effects of the modulation of a coherent TR spectrum by an external magnetic field $[12,13]$ and a uniaxial pressure are presented. These new optical effects are caused by induced optical anisotropy and are realized only for TR with well-pronounced coherent properties.

\section{Some theoretical considerations}

Let us consider a semiconductor plane-parallel layer $(0 \leq z \leq l)$ placed on a substrate $(z>l)$; the surface $z=0$ is contacting with vacuum. We label these media (vacuum, layer, and substrate) with the subscript $j=1,2$, and 3 , respectively. The system is at a temperature $T$. The semiconductor has refractive index $n$ and absorption coefficient $\alpha$. Let $d J=J\left(\lambda, \vartheta_{1}\right) \cos \vartheta_{1} d \lambda d \Omega d S$ be the intensity of radiation in the wavelength range $d \lambda$ from the area $d S$ of the surface $z=0$ into an element $d \Omega$ of the solid angle (oriented at an angle $\vartheta_{1}$ to the normal). According to Kirchhoff's principle, $J\left(\lambda, \vartheta_{1}\right)$ is given by the expression

$J=A\left(\lambda, \vartheta_{1}\right) \frac{2 h c^{2}}{\lambda^{5}(\exp (h c / \lambda k T)-1)}$, 
where $A$ is the radiator emissivity (that is equal to its absorptivity). The absorptivity is defined as a fraction of the radiation energy flux (incident on the surface $z=0$ ) that is absorbed by the system. The quantity $J$ coincides with the blackbody radiation intensity if $A=1$. The polarization properties of TR are described with the partial emissivities $A_{s}$ and $A_{p}$ (and $A=\left(A_{s}+A_{p}\right) / 2$ ). Here, the subscripts $s$ and $p$ label the quantities related to the $s$ - and $p$-polarizations, respectively.

The coefficients $A_{s}$ and $A_{p}$ for the structure considered were calculated in [6]. For a thick $(4 \pi n l / \lambda>>1)$ layer with weak absorption ( $\alpha<<4 \pi n / \lambda)$, one can present the partial emissivities of the structure with a transparent substrate as

$$
A_{v}=\frac{\left(1-R_{1 v}\right)\left(1+\eta R_{2 v}\right)(1-\eta)}{1+2 \eta r_{12 v} r_{23 v} \cos \delta+\eta^{2} R_{1 v} R_{2 v}} .
$$

Here, $v=s, p, R_{1 v}=r_{12 v}^{2}$ and $R_{2 v}=r_{23 v}^{2}$ are the coefficients of reflection from the front (radiating) and back surfaces of the structure, respectively; $r_{12 v}$ and $r_{23 v}$ are the real parts of the Fresnel reflection amplitudes, $\eta=\exp \left(-\alpha l / \cos \vartheta_{2}\right)$ is the transmission factor; $\delta=(4 \pi n l / \lambda) \cos \vartheta_{2}$; and the angle $\vartheta_{2}$ is related to the external angle $\vartheta_{1}$ by the relation $\sin \vartheta_{1}=n \sin \vartheta_{2}$.

For a layer on the absorbing substrate (when the whole structure is non-transparent), one obtains

$$
A_{v}=\frac{\left(1-R_{1 v}\right)\left(1-\eta^{2} R_{2 v}\right)}{1+2 \eta r_{12 v} r_{23 v} \cos \delta+\eta^{2} R_{1 v} R_{2 v}} .
$$

Equations (2) and (3) describe the quickly oscillating dependence of emissivity on the radiation wavelength $\lambda$ at a fixed observation angle $\vartheta_{1}$. For a given $\lambda$, the intensity is an oscillating function of the angle $\vartheta_{1}$. The positions of extrema are determined by the interference conditions

$$
l \cos \vartheta_{2}=\frac{\lambda}{4 n} m,
$$

where $m>1$ are integers: even numbers for peaks and odd ones for minima (if $r_{12 v} r_{23 v}<0$ ), or odd numbers for peaks and even ones for minima (if $r_{12 v} r_{23 v}>0$ ). Conditions (4) coincide with those determining the positions of peaks and minima of the intensity of light passing through a Fabry-Perot interferometer.

Equations (2) and (3) are valid also for a layer with mirrors (dielectric or metal coatings) deposited onto its large faces (these mirrors change the reflection coefficients). In such a structure, due to additional phase jumps at the layer faces, the positions of oscillation peaks differ from those given by Eq. (4). However, the oscillation amplitudes are determined by the same equations. From here on, we refer to the interfaces with reflection coefficients $R_{1}$ and $R_{2}$ as mirrors 1 and 2, respectively.
Let us define the amplitude of the emissivity peaks for each polarization as $\Delta A=A_{\max }-A_{\min }$ (the subscript $v$ is omitted), where $A_{\max }$ and $A_{\min }$ are the emissivity values in the peak and the adjacent minimum, respectively. A TR line corresponds to every emissivity peak. The difference between the intensities in a peak and in an adjacent minimum will be referred to as the line amplitude $\Delta J$. Let us determine the radiation line width $(\Delta \lambda)$ at a half amplitude:

$$
J\left(\lambda_{\max }+\frac{\Delta \lambda}{2}\right)-J_{\min }=\frac{\Delta J}{2} .
$$

Taking into account that $\Delta \lambda<<\lambda_{\max }$ (for thick layers), one obtains from Eq. (5):

$$
\Delta \lambda=\frac{\lambda_{\max }^{2}}{2 \pi n l} \arccos \left[\frac{2 \eta\left(R_{1} R_{2}\right)^{1 / 2}}{1+\eta^{2} R_{1} R_{2}}\right] .
$$

One can see from Eq. (6) that an increase of the parameter $\eta\left(R_{1} R_{2}\right)^{1 / 2}$ leads to the narrowing of the radiation line width.

We also studied how the TR spectrum depends on some external actions such as a magnetic field and a uniaxial deformation. Let us consider TR emitted along a normal to the layer in a constant magnetic field $\mathbf{B}=$ $(0,0, B)$ (the Faraday configuration). Inside the layer, the normal waves are circularly polarized waves with complex refractive indices $n_{ \pm}=\left(\varepsilon_{x x} \mp i \varepsilon_{x y}\right)^{1 / 2}$. The labels "+" and "_" are referred to waves with the right and left polarizations, respectively; the components of the dielectric tensor $\varepsilon_{\alpha \beta}(\omega, B)$ obey the conditions $\varepsilon_{x x}=\varepsilon_{y y}, \varepsilon_{x y}=-\varepsilon_{y x}$.

In order to explain the magnetic field effect more clearly, we do not use Kirchhoff's law but obtain the spectral intensity of TR from a direct calculation. We assume that the layer radiates due to thermal fluctuations of the electric current density [14]. For the TR intensity, we use Eq. (1), where now

$A=\left(A_{+}+A_{-}\right) / 2$.

Here, $A_{ \pm}$is the plate emissivity attributed to the mode " \pm ". Note that $A(B)=A(-B)$ and the emissivity coincides with the layer absorptivity. Hence, the intensity obtained obeys Kirchhoff's law.

The spectral intensity of radiation is determined by the sum of the partial intensities attributed to the "+" and "_" modes, and the interference conditions (4) are valid for each mode. Since $n_{+} \neq n_{-}$, the positions of the extrema in the " \pm " spectra generally do not coincide, and this may lead to a "beat effect" resulting in a lowfrequency modulation of the total spectrum.

The effect of a low-frequency modulation of the TR spectrum by an external magnetic field is analogous, in its nature, to that observed in plane-parallel plates with anisotropic (due to a uniaxial strain) permittivity. In 
the plate $(0 \leq z \leq l)$ of a uniaxial crystal with the optical axis oriented along the $x$-axis, the light waves propagating along the $z$-axis are the two waves with different indices of refraction: $n_{\|}$and $n_{\perp}$ for the waves with the electric vectors $\mathbf{E}=(E, 0,0)$ and $\mathbf{E}=(0, E, 0)$, respectively. In the linear (in the strain) approximation,

$n_{\perp}=n+\gamma P, \quad n_{\|}=n-\gamma \sigma P$.

Here, $\gamma$ is a phenomenological coefficient, $P$ pressure, and $\sigma$ the Poisson's ratio.

The polarizations of these waves are mutually perpendicular, and the interference between them does not occur. Hence, the TR spectrum is the sum of the partial spectra attributed to each type of waves, and a low-frequency modulation of the total spectrum can take place.

\section{Experimental results}

In our experimental studies of TR, we used the planeparallel plates of $n$-GaAs (the concentration of uncompensated donors $\left.N_{d}-N_{a}=1 \times 10^{18} \mathrm{~cm}^{-3}\right), n-\mathrm{Si}$ $\left(N_{d}-N_{a}=6 \times 10^{17} \mathrm{~cm}^{-3}\right.$ and $\left.6 \times 10^{16} \mathrm{~cm}^{-3}\right), n$-InAs $\left(N_{d}-\right.$ $\left.N_{a}=1.4 \times 10^{18} \mathrm{~cm}^{-3}\right)$ single crystals and the $\mathrm{Pb}_{0.8} \mathrm{Sn}_{0.2} \mathrm{Te} / \mathrm{BaF}_{2} \quad$ films of $p$-type $\left(N_{a}-N_{d}=\right.$ $\left.2 \times 10^{18} \mathrm{~cm}^{-3}\right)$. The plane-parallel plates were fabricated using a mechanical grinding with a subsequent polishing of their broad faces. The deviation from planeparallelism was estimated from the transmission spectra. The plates with the biggest difference of intensities in the interference maxima and minima of the transmission spectra were chosen for the measurements. The plates, for which the interference effect was negligible (e.g., wedge-shaped plates), were used in our experiments for comparison. In what follows, we refer to these samples as non-plane-parallel plates.

The plates had lateral dimensions of about $10 \times 15 \mathrm{~mm}$ and thickness $\approx 100 \mu \mathrm{m}$. The thickness of the films obtained by laser evaporation [15] was $1.1 \mu \mathrm{m}$. The measurements were performed in the spectral region of free charge carrier absorption using a Fourierspectrometer with a spectral resolution of $1 \mathrm{~cm}^{-1}$. The aperture of the optical equipment inlet did not exceed $2.5^{\circ}$. The intensity of radiation was normalized to the blackbody radiation measured under the same experimental conditions.

\subsection{TR angular distributions}

The angular distributions of the TR from the planeparallel Si plate $\left(N_{d}-N_{a}=6 \times 10^{17} \mathrm{~cm}^{-3}\right)$ are shown in Figs. 1a and b. The solid curves show the calculated ratios between the emissivities $A_{v}\left(\vartheta_{1}\right)$ and $\overline{A_{v}}\left(\vartheta_{1}\right)$ of the plane-parallel and non-plane-parallel plates, respectively, with different directions of polarizations $(\lambda=10.57 \mu \mathrm{m})$. One can see that, because of the multiple-beam interference, the radiation directional pattern has a number of pronounced lobes. The angular dependences $A_{v}\left(\vartheta_{1}\right) / \bar{A}_{v}\left(\vartheta_{1}\right)$ for the $s$ - and $p$-polarized emission (Fig. 1b and the left-hand side of the pattern in Fig. 1a) differ markedly from each other. In the $55^{\circ}-75^{\circ}$ range, the extrema for the $p$-polarized radiation virtually disappear; this is related to a drop (almost to zero) of the reflectance $R_{p}$. The angular dependences for the $s$ polarized and unpolarized emissions are similar in many respects, see Fig. 1a. One can see that the calculated and experimental data are in good agreement (in our calculations, we took the dependences $\alpha(\lambda)$ and $n_{2}(\lambda)$ obtained from our measurements into account).
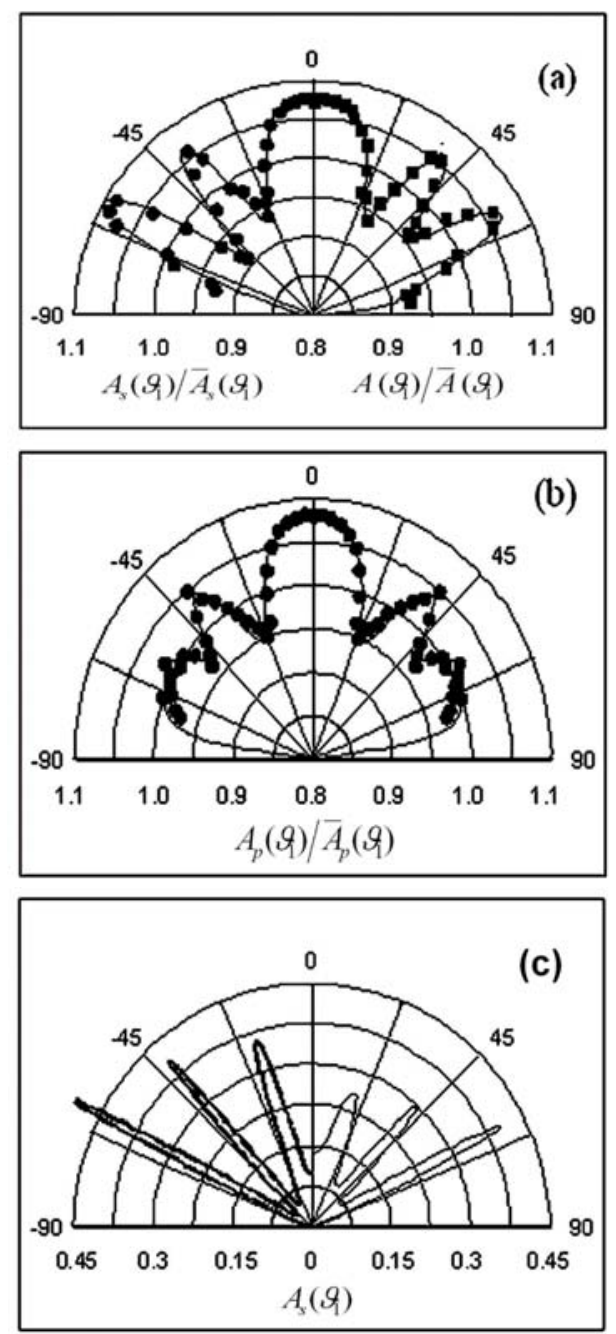

Fig. 1. Angular distributions of TR from $n$-Si plane-parallel plates for $\lambda=10.57 \mu \mathrm{m}$ and $l=92 \mu \mathrm{m}$. The solid lines correspond to the results of calculation, while the dots correspond to the experimental results. (a) and (b) - the ratios between the emissivities of the plane-parallel and identical non-plane-parallel plates; (c) - the emissivities of planeparallel plates. (a): the left-hand side corresponds to $s$ polarization; the right-hand side, to unpolarized radiation. (b) corresponds to $p$-polarization. (c): to $s$-polarization. The parameters used in the calculation: $(a, b)-\eta=0.93, n=3.4$; (c) - plates with $l=179 \mu \mathrm{m}$ and $n=3.4$ (right-hand side), $n=6$ (left-hand side of the pattern), $\eta=0.87$. 
A more pronounced TR pattern can be obtained in the angular dependences if $R_{1,2}$ of the plane-parallel plate are increased. This effect can be seen from Fig. 1c that shows the calculated angular dependences for the emissivities $A_{s}\left(\vartheta_{1}\right)$ of a plate with the refractive index $n=6$ (the left-hand side of the pattern) and that with $n=$ 3.4 (the right-hand side of the pattern). For both plates, $l=179 \mu \mathrm{m}$ and $\eta=0.87$ (for $\vartheta_{1}=0$ ).

\subsection{TR spectral dependences}

Oscillating curve 1 in Fig. 2 shows the spectral dependence (measured at the angle $\vartheta_{1}=60^{\circ}$ to the normal) of the TR intensity for a plane-parallel $n$-GaAs plate with an Al layer on its back face. (In this case, $s$ polarization is considered.) One can see that TR from a plane-parallel plate is drastically modified as compared to that from a blackbody (curve 2). The radiation intensity in peaks practically approaches that of a blackbody.

Figure 3 shows the experimental spectral dependences for the emissivities of the plane-parallel GaAs plate (curve 1) and the non-plane-parallel plate (steadily increasing curve 2). Curve 3 shows the measured variance of the optical thickness al for the plane-parallel GaAs plate. The oscillations are pronounced better in the wavelength area where absorption is weak. For $\lambda>14 \mu \mathrm{m}$, when absorption becomes strong $(\alpha l>2.5$, see Fig. 3, curve 3), the multibeam interference is suppressed, and the oscillations practically vanish in the noise. One can see that the maxima and minima of the emission from the plane-parallel plate are arranged virtually symmetrically relative to the curve of TR from the non-plane-parallel plate.

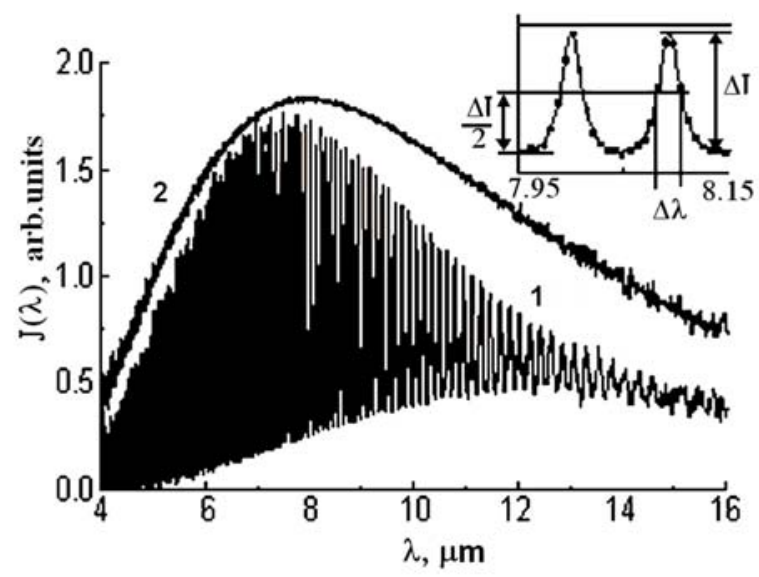

Fig. 2. Spectral dependence of $s$-polarized TR: 1 - from a plane-parallel $n$-GaAs plate with an Al layer on its back face. The viewing angle $\vartheta_{1}=60^{\circ}, R_{1 S} \approx 0.51, T=365 \pm 2 \mathrm{~K}$. $2-$ from a blackbody under the same experimental conditions. The inset shows the same spectra in the $\lambda=7.95-8.15 \mu \mathrm{m}$ area. Marks experimental $J(\lambda)$ values, the curve - the result of the fitting to the experimental values.
The curves in Fig. 4 present the emissivity of a thin $\mathrm{Pb}_{0.8} \mathrm{Sn}_{0.2}$ Te film; TR is emitted along the normal to the film surface. In the calculation of $A(\lambda)$, we took the dispersion of $\alpha$ and $n$ in the film into account that was determined from our independent measurements (for $\mathrm{Pb}_{0.8} \mathrm{Sn}_{0.2}$ Te films, $n \approx 6$ ). Note that the thick substrate $(L=1.5 \mathrm{~mm})$ with high transparency and small refraction coefficient $\left(n_{3} \approx 1.4\right)$ contributed practically nothing to TR. One can see from Figs. 2 and 4 that a good agreement is obtained between the measurements and the theory for both thick $(\lambda / n<<l$, plates $)$ and thin $(\lambda / n \approx l$, films) layers.

\subsection{The amplitudes of TR lines}

To investigate the dependence of the amplitudes of emissivity peaks on the coefficients of absorption and reflection, we chose $n$-GaAs (the concentration of uncompensated donors $N_{d}-N_{a}=1.1 \times 10^{18} \mathrm{~cm}^{-3}$ ). Its absorption coefficient beyond the fundamental

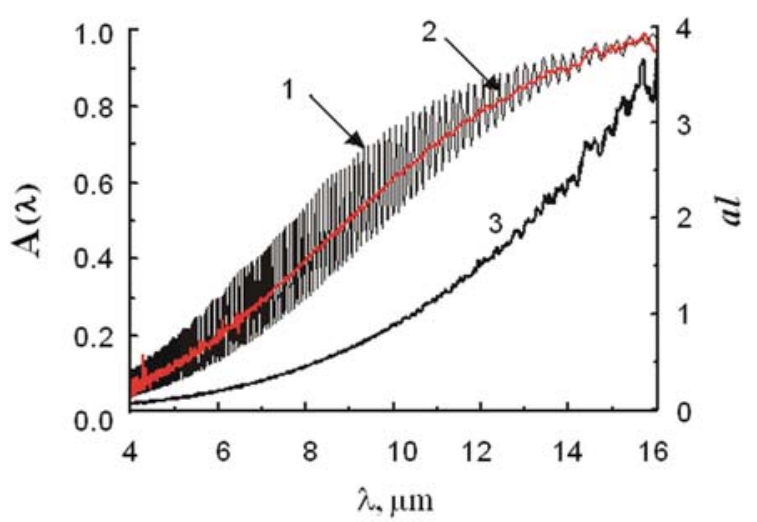

Fig. 3. Experimental spectral dependences of emissivity $A(\lambda)$ (curves 1,2 ) and optical thickness $\alpha l$ (curve 3) for the $n$ GaAs plate; 1 is a plane-parallel plate, and 2 is the identical non-plane-parallel one. The viewing angle $\vartheta_{1}=0^{\circ}$.

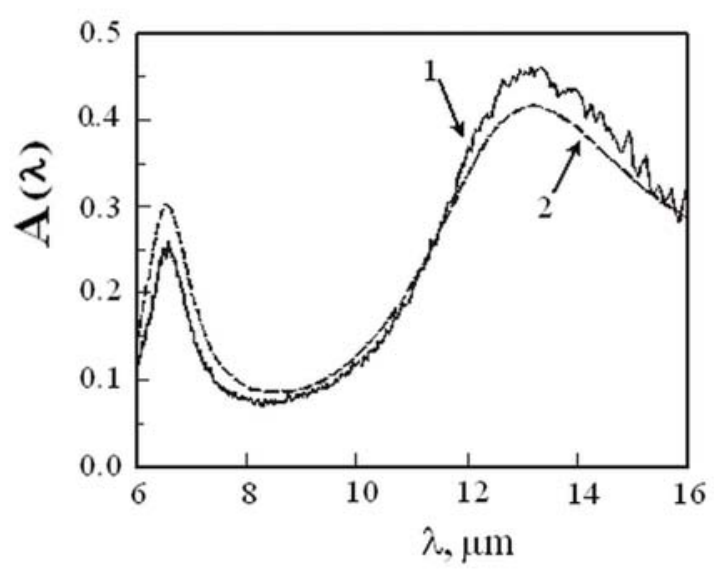

Fig. 4. Spectral emissivity of a $\mathrm{Pb}_{0.8} \mathrm{Sn}_{0.2} \mathrm{Te}$ film $(l=1.1 \mu \mathrm{m})$. 1 - experiment, 2 - theory. 
absorption edge is characterized by strong dispersion: $\alpha(\lambda) \propto \lambda^{3}$ [16]. This fact ensures the variation of the transmission factor $\eta(\lambda)$ from $\eta \approx 0.98$ down to $\eta \approx 0.01$ in the spectral range $3-17 \mu \mathrm{m}$ used by us. One can follow the variation of the radiation line amplitude in a wide transmission range by presenting the radiation spectrum as a function of $\eta(\lambda)$.

The curves in Fig. 5 present the theoretical and experimental dependences of the amplitudes of emissivity peaks, $\Delta A$, on the transmission factor $\eta$ at different mirrors on the faces. Figure 5a demonstrates $\Delta A(\eta)$ for a symmetric structure with non-absorbing mirrors $\left(R_{1}=R_{2}\right)$. The asymmetric cases - the plates with absorbing (e.g., metal) mirror 2 and non-absorbing mirror 2 - are illustrated by Figs. $5 \mathrm{~b}$ and c, respectively.

One can see that the $\Delta A(\eta)$ curves are nonmonotonic and have a peak, whose position $\left(\eta_{\max }\right)$ and height $\left(\Delta A_{\max }\right)$ depend on the reflection value. The $\Delta A_{\max }$ value grows and shifts towards the higher transparency region as the coefficient of reflection from the radiating face increases.

This maximum is achieved at different values of the transmission factor, depending on the reflection from faces. One can see that $\eta_{\max }$ increases with $R_{1}$ and $R_{2}$. If good resonator conditions in a plate are ensured, then the radiation redistribution over wavelengths is so strong that the radiation line amplitude approaches the blackbody radiation intensity.

However, one can see from Fig. $5 b$ that, in the case of a sample with an absorbing mirror at its back side, $\Delta A(\eta)$ does not tend to zero as $\eta \rightarrow 1$. A nonzero value of $\Delta A$ at $\eta=1$ is due to the additional illumination from the non-transparent substrate (absorbing mirror) on the back surface. The substrate emissivity is small: in our case, $1-R_{2}=0.05$ for curve 3 in Fig. $5 b$ and 0.19 for curves 1 and 2 in Fig. 5b. However, its contribution to TR is considerable. Under sufficiently strict resonator conditions, just this radiation may become predominant in the total radiation from the structure. One can see from curve 3 in Fig. $5 b$ that the amplitude of radiation lines for an absolutely transparent $(\eta=1)$ structure with an absorbing mirror $\left(R_{2}=0.95\right)$ and a nonabsorbing mirror at the radiating face $\left(R_{1}=0.94\right)$ practically approaches the intensity of blackbody radiation. It should be noted that curve 3 in Fig. $5 \mathrm{~b}$ is also nonmonotonic. Its peak at $\eta \approx 1$ is pronounced very weakly and is not seen on the plot.

If non-absorbing mirrors are applied, then there is no additional illumination effect, see Fig. 5c. Such mirror coatings can be realized using an interference multilayer structure made of IR-transparent materials. The $\Delta A(\eta)$ curves for non-symmetric structures are qualitatively similar to those in the symmetric case (Fig. 5a). However, at high $R_{2}$ value in a non-symmetric structure, the amplitude of spectral lines is considerably higher and may reach that for a blackbody.
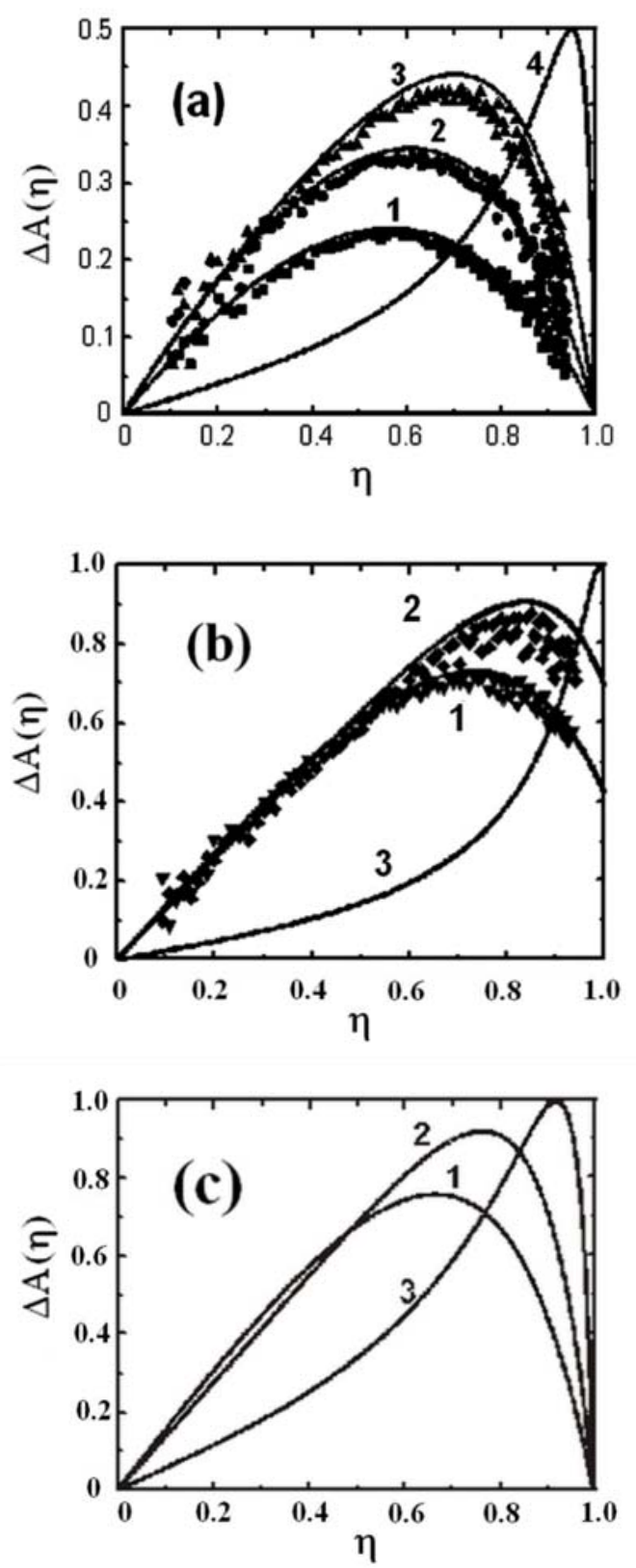

Fig. 5. Dependences of the emissivity peak amplitudes $\Delta A$ on the transmission factor $\eta$ for plane-parallel plates with different coefficients of reflection from the front (radiating) $\left(R_{1}\right)$ and back $\left(R_{2}\right)$ faces. Curves - the results of calculation. Marks the results of analysis of the experimental spectra of $s$ polarized TR from $n$-GaAs plates $\left(N_{d}-N_{a}=1.1 \times 10^{18} \mathrm{~cm}^{-3}, l=\right.$ $100 \mu \mathrm{m}$ ) at different viewing angles $\vartheta_{1} \cdot T=365 \pm 2 \mathrm{~K}$. (a) mirrors 1 and 2 are non-absorbing, $R_{1}=R_{2} .1-R_{1}=0.27 ; 2-$ $R_{1}=0.43 ; 3-R_{1}=0.64 ; 4-R_{1}=0.95$. Marks - a free $n$-GaAs plate, $R_{1 S}=R_{2 S}:-\vartheta_{1}=0^{\circ}, R_{1 S} \approx 0.27 ; \bigcirc-\vartheta_{1}=50^{\circ}, R_{1 S} \approx 0.43$; $\boldsymbol{\Delta}-\vartheta_{1}=70^{\circ}, R_{1 S} \approx 0.64$. (b) - mirror 1 is non-absorbing, mirror 2 is absorbing. $1-R_{1}=0.27, R_{2}=0.81 ; 2-R_{1}=0.52, R_{2}=$ $0.81 ; 3-R_{1}=0.94, R_{2}=0.95$. Marks - an $n$-GaAs plate with an Al layer on the back face, $R_{2}=0.81$. $\nabla-\vartheta_{1}=0^{\circ}, R_{1 S} \approx 0.27$; $\checkmark-\vartheta_{1}=60^{\circ}, R_{1 S} \approx 0.52$. (c) - mirrors 1 and 2 are non-absorbing, $R_{2}=1.1-R_{1}=0.27 ; 2-R_{1}=0.65 ; 3-R_{1}=0.85$. 
The $\Delta A(\eta)$ dependences (obtained from the analysis of the experimental TR spectra for planeparallel GaAs plates) are shown in Figs. $5 \mathrm{a}$ and $\mathrm{b}$ by marks. On the abscissa, the mean $\eta$ value in the $\lambda_{\max }-\lambda_{\min }$ spectral range is plotted for each mark. Since the $\eta$ variation in those ranges did not exceed $6 \%$, one can conclude that the error of such a representation is insignificant. When calculating the $J(\eta)$ dependence from the experimental spectrum $J(\lambda)$, we used the dispersion dependence $\eta(\lambda)$ that was determined by us experimentally for the given material from its transmission and reflection spectra.

Figure 5a demonstrates the symmetric case (the reflection coefficients at both faces are the same). The GaAs plate surfaces were clean, and the $R_{1}$ and $R_{2}$ values were varied by changing the angle of outgoing radiation viewing. In Fig. 5b, we show the $\Delta A(\eta)$ dependences for an $n$-GaAs plane-parallel plate with an aluminum mirror deposited onto the back face. In this case, the coefficient of reflection from the back face was $R_{2}=0.81$ (this value was determined by us experimentally).

The curve in Fig. 6 presents the dependence of the relative radiation line width $\left(\Delta \lambda / \lambda_{\max }^{2}\right)$ on the parameter $\eta\left(R_{1} R_{2}\right)^{1 / 2}$. The marks show the $\Delta \lambda / \lambda_{\max }^{2}$ values determined from the experimental TR spectra for the $n$-GaAs plates (with free faces and with an aluminum mirror on the back face).

We determined $\Delta \lambda$ for radiation lines in a narrow spectral range $(\lambda=8-9 . \mu \mathrm{m})$, where $n$ varies insignificantly (in our calculations, we used the $n$ value averaged over the above spectral range: $n=3.18$ ). When analyzing the line parameters, we applied the fitting to the experimental results. There is a good agreement between the experimental results and those of theoretical calculations.

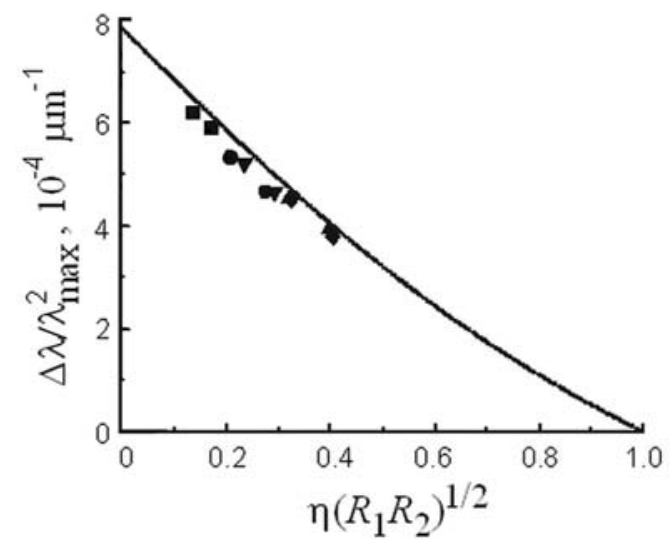

Fig. 6. Dependence of the relative radiation line width $\Delta \lambda / \Delta \lambda^{2}{ }_{\text {max }}$ on the parameter $\eta\left(R_{1} R_{2}\right)^{1 / 2} ; n_{2}=3.18 ; l=100 \mu \mathrm{m}$. The curve is the result of calculation. Marks are the results of analysis of experimental data in the $\lambda=8-9 \mu \mathrm{m}$ range: $\mathbf{\square}_{-}$ $\vartheta_{1}=0^{\circ}, R_{1}=R_{2} \approx 0.27 ; \bigcirc-\vartheta_{1}=50^{\circ}, R_{1 S}=R_{2 S} \approx 0.43 ; \boldsymbol{\Delta}-$ $\vartheta_{1}=70^{\circ}, R_{1 S}=R_{2 S} \approx 0.64 ; \boldsymbol{\nabla}-\vartheta_{1}=0^{\circ}, R_{1} \approx 0.27, R_{2}=0.81$; $\diamond-\vartheta_{1}=60^{\circ}, R_{1 S} \approx 0.52, R_{2}=0.81$.

\subsection{Influence of a magnetic field on the TR spectrum}

For the measurements of spectra, the plane-parallel plates made of $n$-InAs were used (this material is isotropic in the absence of a magnetic field). The high concentration of free electrons $\left(N_{e}=1.4 \times 10^{18} \mathrm{~cm}^{-3}\right)$ made it possible to carry out the measurements in a classical magnetic field: the Landau splitting energy $\hbar \omega_{c}$ is small as compared with $k T\left(\omega_{c}\right.$ is the cyclotron frequency). The $10 \times 13 \mathrm{~mm}$ samples had the thickness $l=100 \mu \mathrm{m}$; the deviation from plane-parallelism was no more than a few seconds of arc.

The plate was placed between the magnet poles so that the magnetic field was directed normally to the broad faces of the sample, and TR emitted along the magnetic field was measured.

In Fig. 7, we present the experimental TR spectra taken without magnetic field (Fig. 7a) and in the magnetic field $B=1.3 \mathrm{~T}$ (Fig. 7e). In the magnetic field, a bottleneck appears at nearly $\lambda \approx 9.2 \mu \mathrm{m}$ where the interference extrema practically disappear. As the wavelength grows, the interference becomes apparent again; the spectrum extrema, however, are in antiphase, as compared with those at $B=0$.

The polarization properties of TR were demonstrated by its transformation into the linear polarized radiation. For this purpose, we used a Ge total internal reflection prism with base angles of $42^{\circ} 56^{\prime}$. Since the polarization planes of right- and left-hand polarized radiation are mutually perpendicular, we separated the required radiation mode by applying a polarizer-analyzer.

Figures $7 \mathrm{~b}$ and $7 \mathrm{c}$ show the spectra of the right- and left-hand circular polarized modes of TR, respectively. Since $n_{+} \neq n_{-}$, the positions of extrema of the spectrum in Figs. $7 \mathrm{~b}$ and $7 \mathrm{c}$ generally do not coincide. Such a difference in the partial spectra leads to a "beat effect" resulting in a low-frequency modulation of the total spectrum. One can see that the oscillation phase of the right-hand circularly polarized mode takes the lead over that of the left-hand circularly polarized mode, and they are in antiphase at $\lambda \approx 9.2 \mu \mathrm{m}$. The oscillations in the total spectrum (recorded without a polarizer-analyzer, Fig. 7e) are not observed practically in this wavelength range. In Fig. $7 d$, we give the arithmetic sum of the spectra of the right-hand and left-hand circularly polarized modes; it agrees well with the total TR spectrum shown in Fig. 7e. This result proves that the mode "+" ("-") had a right-hand (left-hand) circular polarization in our experiments. Some insignificant distinction between the spectrum amplitudes in Figs. 7e and $\mathrm{d}$ is due to the losses introduced by a polarizeranalyzer. The theoretical spectra agree well with the experimental ones for the electron effective mass $m=0.04 m_{e} \quad$ (this value was determined by the comparison and adjustment of the calculated and measured spectra; it is in good agreement with the known data [17]). 


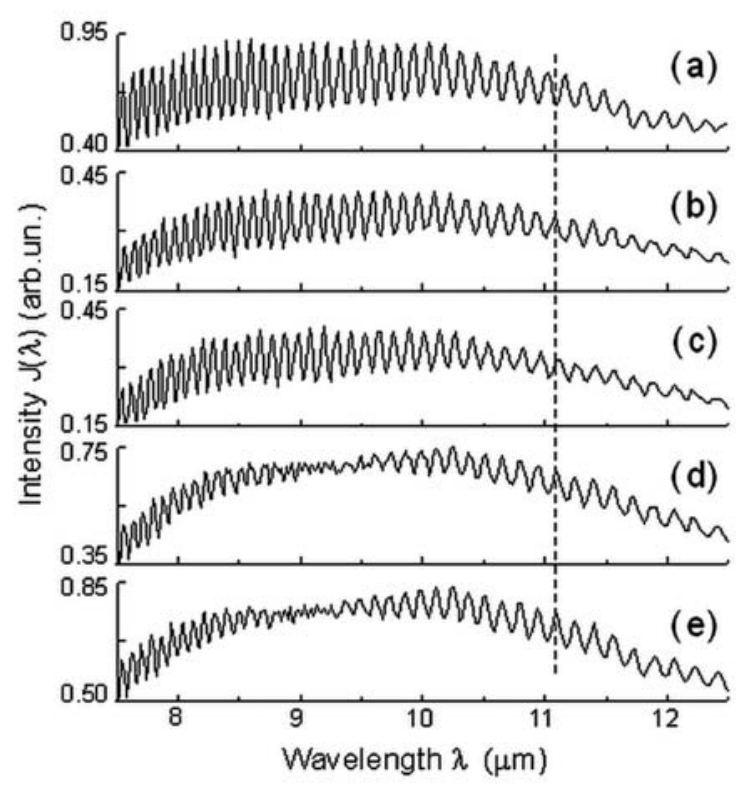

Fig. 7. Experimental spectra of TR from an $n$-InAs plate $\left(N_{e}=\right.$ $\left.1.4 \times 10^{18} \mathrm{~cm}^{-3}, \quad l=100 \mu \mathrm{m}, \quad T=375 \mathrm{~K}\right)$ without magnetic field (a) and in the magnetic field $B=1.3 \mathrm{~T}$. (b) - right-hand circularly polarized mode; $(\mathrm{c})$ - left-hand circularly polarized mode; (d) - a sum of (c) and (d) spectra; (e) - the spectrum recorded without analyzer.

It is worth to note that the reported effect appears in the radiation of intrinsic unpolarized light and thus differs in principle from the known magnetooptical effects such as the Faraday and Voigt effects or magnetoplasma reflection that describe the peculiarities of the transmission (or reflection) of external polarized light. It should be emphasized that the effect under consideration is realized for TR with well-pronounced coherent properties only.

\subsection{Influence of uniaxial deformation on the $T R$ spectrum}

When studying the TR and the transmission spectra of uniaxially compressed plane-parallel plates, we used $\mathrm{Si}$ with $N_{d}-N_{a}=6 \times 10^{17} \mathrm{~cm}^{-3}$ and $6 \times 10^{16} \mathrm{~cm}^{-3}$, respectively. The $5 \times 8 \mathrm{~mm}$ crystals (the thickness $l=350 \mu \mathrm{m}$ ) were compressed along their long face (i.e., along the [100] axis). We investigated the radiation propagating along a normal to the direction of compression, i.e., to the optical axis. The optical surfaces of the sample coincided with the (100) plane. In this case, the waves with the electric vector oriented along and perpendicularly to the optic axis have different velocities related to the refractive indices $n_{\|}$and $n_{\perp}$, respectively. Therefore, the radiation leaving a uniaxially strained crystal is a superposition of two oscillating interference spectra, each of them corresponding to $n_{\perp}$ or $n_{\|}$.

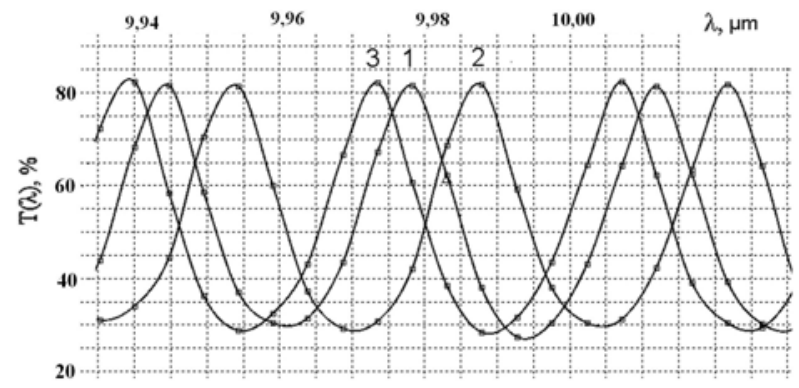

Fig. 8. Experimental transmission spectra of a uniaxially strained plane-parallel Si sample. $1-P=0$, arbitrary direction of the polarization plane of the detected radiation; $2-P=$ $6 \mathrm{kbar}$, the polarization plane is perpendicular to the direction of compression; $3-P=6 \mathrm{kbar}$, the polarization plane is parallel to the direction of compression. $T=355 \mathrm{~K}$.

The samples were strained uniaxially in a special facility; it ensured a mechanical stress that was uniform over the sample cross section. The transmission spectra were recorded when the sample was illuminated with unpolarized radiation. Investigations of both TR and transmission spectra were carried out in two ways, namely, with and without an analyzer between the sample under investigation and a spectrometer receptor. The analyzer (a grating-polarizer) was placed so that its axis was oriented either in parallel to the compression direction or along a normal to it. The TR and transmission spectra were measured in the $3-18-\mu \mathrm{m}$ wavelength range; the sample temperature was $T=$ $355 \mathrm{~K}$.

In Fig. 8, we display the transmission spectra (in the wavelength range $9.935-10.03 \mu \mathrm{m}$ ) of an unstrained (curve 1) and strained (pressure $P=6 \mathrm{kbar}$ ) samples taken when the analyzer axis was perpendicular to the compression direction (curve 2) and in parallel to it (curve 3). (At $P=0$, the crystal is isotropic, so curve 1 is repeated at any of the above analyzer orientations.) The form of the spectra taken is typical of the transmission interference spectra of plane-parallel samples. The positions of maxima and minima in each spectrum are determined by the refractive index $n$ (curve 1), $n_{\perp}$ (2), and $n_{\|}$(3), respectively. The spectral shifts of curves 2 and 3 relative to curve 1 determine birefringence ( $n_{\perp}-n_{\|}=6 \times 10^{-3}$ at $P=6 \mathrm{kbar}$ ). We note that the TR spectra are qualitatively similar to those of transmission because the positions of maxima and minima in both spectra of a plane-parallel plate are just the same if the measurements are made at the same temperature.

The spectra of transmission of unpolarized light and TR taken without an analyzer demonstrate the presence of beats (low-frequency amplitude modulation). Figure 9 presents the calculated emissivity spectrum of an uniaxial deformed $\mathrm{Si}$ sample; the parameters are as follows: $l=350 \mu \mathrm{m}, \quad n=3.4$, $n_{\perp}=3.4018, \quad n_{\|}=3.3990 \quad(P=3 \mathrm{kbar}) \quad$ and 
$n_{\perp}=3.4025, n_{\|}=3.3986(P=4 \mathrm{kbar})$, the dependence $\alpha(\lambda)$ was not taken into account. The values of $n_{\perp}$ and $n_{\|}$were obtained from an analysis of the experimental
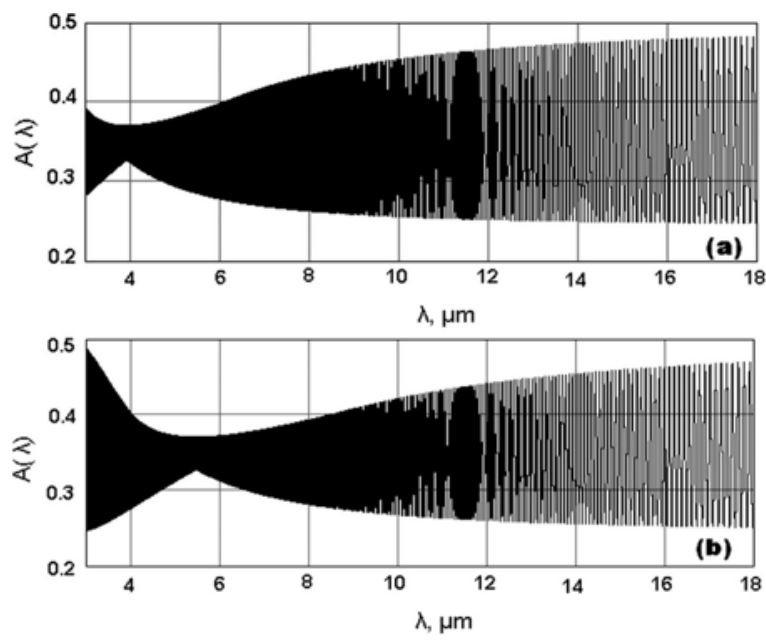

Fig. 9. Calculated emissivity spectra of a uniaxially compressed $\mathrm{Si}$ plane-parallel plate. $P=3 \mathrm{kbar}(\mathrm{a})$ and $4 \mathrm{kbar}(\mathrm{b}) ; T=355 \mathrm{~K}$.
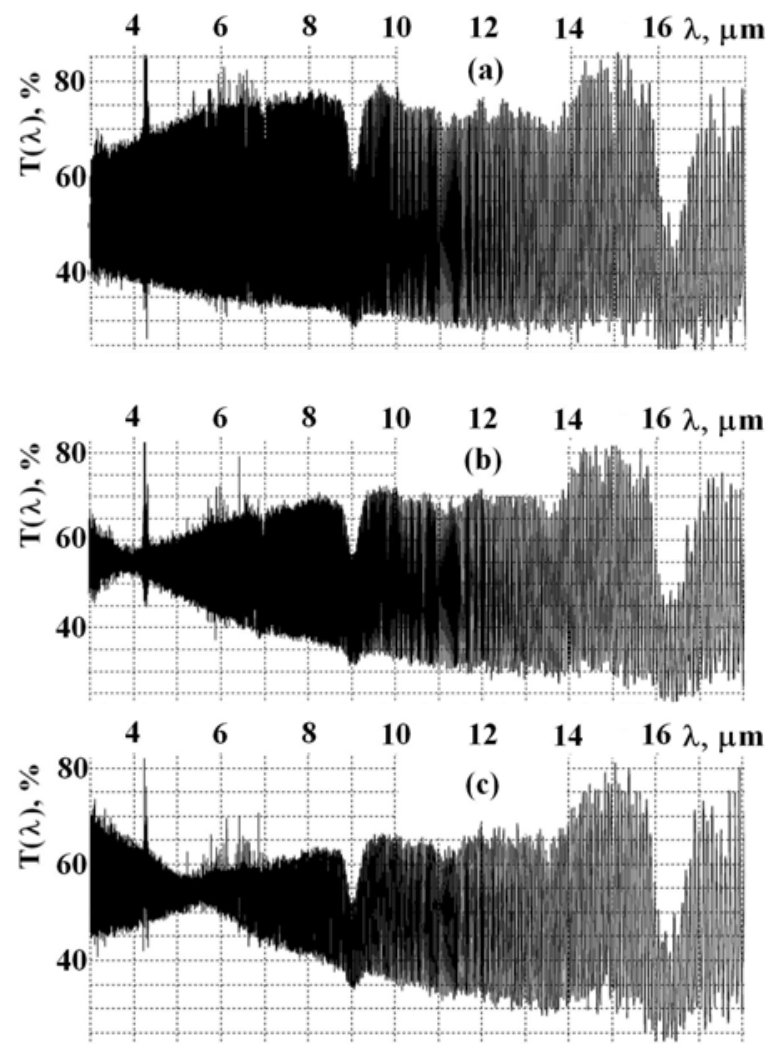

Fig. 10. Experimental transmission spectra of a uniaxially compressed plane-parallel $\mathrm{Si}$ sample (no analyzer). $P=0$ (a), 3 (b), and 4 kbar (c); $T=355 \mathrm{~K}$. transmission spectra. They correspond to those given by Eq. (8) at $\gamma=7.3 \times 10^{-7}$ bar $^{-1}$ and $\sigma=0.3$. One can see that the beat node shifts towards a low-energy part of the spectrum as the pressure $P$ (and, with it, the difference $\left.n_{\perp}-n_{\|}\right)$increases. The experimental transmission spectrum is shown in Fig. 10 (the spectral bands near 9 and $16 \mu \mathrm{m}$ are the regions of absorption induced by the oxygen vibration absorption and the lattice absorption, respectively). The position of the beat node, as well as its spectral shift, is in close agreement with the calculated data.

It should be noted that, as far as we know, the lowfrequency modulation in the transmission spectra of anisotropic resonator objects has not been observed before, and we seem to be the first to report it.

The investigation of the TR spectra under the uniaxial compression, as well as under the action of an external magnetic field, makes it possible to determine the parameters of semiconductors at high temperatures.

\section{Conclusions}

In this paper, we have investigated the dependence of coherent TR of semiconductor planar resonator structures on their optical parameters, as well as the spectrum modulation of coherent TR by an external magnetic field and a uniaxial pressure.

Due to the multibeam interference, a smooth spectral dependence of radiation is transformed into an oscillating one with sharp peaks. In addition, the angular dependence of TR does not obey Lambert's law and demonstrates a nonmonotonic character with clearly pronounced lobes. We determined the optimal conditions under which the intensity of TR of the planar resonator structures may approach that of blackbody radiation.

The results may be of use when developing new thermal sources of IR radiation, whose operating spectral band is given by the emitting element parameters. When designing a controllable source or optical sensor involving planar resonator structures as a radiating element, one should take into account both the reflection from interfaces and the absorption in emitting layers. The optical parameters and hence the characteristics of the radiating element can be modulated with the help of the known methods of variation of the free charge carrier concentration (e.g., by the contact injection or exclusion, photogeneration, or the magnetoconcentration effect).

We have demonstrated a new magneto-optical effect - the low-frequency modulation of a rapidly oscillating spectrum of TR by an external magnetic field. For the first time, we have investigated TR of anisotropic crystals. We have shown that the lowfrequency modulation appears in the emission (transmission) spectra of uniaxially deformed crystals.

The results obtained will serve to widen the knowledge of emissive properties of different planeparallel objects (say, various films, layers, and coatings). 
They may be applied to the monitoring of layer parameters and the proper account of heat exchange and heat transfer as well.

\section{References}

1. P.J. Hesketh, J.N. Zemel, B. Gerbhart, Organ pipe radiant modes of periodic micromachined silicon surfaces // Nature (London) 324, p. 549-551 (1986).

2. J.-J. Greffet, R. Carminati, K. Joulain, J.-P. Mulet, S. Mainguy, Yong Chen, Coherent emission of light by thermal sources // Nature (London) 416, p. 61-64 (2002).

3. F. Marquier, K. Joulain, J.-P. Mulet, R. Carminati, J.-J. Greffet, Y. Chen, Coherent spontaneous emission of light by thermal sources // Phys. Rev. B 69, 155412 (2004).

4. V.S. Pekar, On the theory of spontaneous and stimulated emission of electromagnetic waves in one-dimensionally inhomogeneous media and resonators // Zhurnal Experiment. Teor. Fiziki 67, p. 471 (1974) (in Russian); Some features of the optical spectra of thin layers // Ibid. 88, p. 667 (1985) (in Russian).

5. A.I. Liptuga, N.B. Shishkina, Thermal radiation of semitransparent plane-parallel objects // Infrared Physics and Technology 44, p. 85-89 (2003).

6. A.G. Kollyukh, A.I. Liptuga, V.A. Morozhenko, V.I. Pipa, Thermal radiation of plane-parallel semitransparent layers // Opt. Communs 225, p. 349-352 (2003).

7. K.Yu. Guga, O.G. Kollyukh, A.I. Liptuga, V.O. Morozhenko, V.I. Pipa, Features of thermal radiation of plane-parallel semiconductor wafers // Semiconductors 38, p. 507-511 (2004).
8. O.G. Kollyukh, A.I. Liptuga, V. Morozhenko, V.I. Pipa, Effect of the optical characteristics of semiconductor resonator structures on the amplitudes of their thermal radiation lines // Opt. Communs 260, p. 607-613 (2006).

9. B.J. Lee, Z.M. Zhang, Design and fabrication of planar multilayer structures with coherent thermal emission characteristics // J. Appl. Phys. 100, 063529 (2006).

10. B.J. Lee, C.G. Fu, Z.M. Zhang, Coherent thermal emission from one-dimensional photonic crystals // Appl. Phys. Lett. 87, 071904 (2005).

11. M. Laroche, R. Carminati, J.-J. Greffet, Coherent thermal antenna using a photonic crystal slab // Phys. Rev. Lett. 96, 123903 (2006).

12. O.G. Kollyukh, A.I. Liptuga, V.A. Morozhenko, V.I. Pipa, Magnetic-field modulation of the spectrum of coherent thermal radiation of semiconductor layers // Phys. Rev. B 71, 073306 (2005).

13. O.G. Kollyukh, A.I. Liptuga, V. Morozhenko, V.I. Pipa, E.F. Venger, Circular polarized coherent thermal radiation from semiconductor layers in an external magnetic field // Opt. Communs 276, p. 131-134 (2007).

14. S.M. Rytov, Y.A. Kravtsov, V.I. Tatarskii, Principles of Statistical Radiophysics, Vol. 3: Elements of Random Fields. Springer-Verlag, Berlin, 1989.

15. S.V. Plyatsko, Yu.S. Gromovoj, F.F. Sizov, Narrow gap IV-VI thin films produced by quasicontinuous-wave $\mathrm{CO}_{2}$-laser-induced evaporation // Thin Solid Films 218, p. 151-156 (1992).

16. R.K. Willardson, A.C. Beer (Eds.), Semiconductors and Semimetals, vol. 3. Academic Press, New York, 1967.

17. O. Madelung, Semiconductors: Data Handbook. Springer, Berlin, 2004. 